\title{
Theory of Evolutionary Algorithm: A View from Thermodynamics
}

\author{
Yuanxiang $\mathrm{Li}^{1}$, Weiwu Wang ${ }^{1}$, Xianjun Shen ${ }^{2}$, Weiqin Ying ${ }^{1}$, and Bojin Zheng ${ }^{3}$ \\ ${ }^{1}$ State Key Lab of Software Engineering \\ Wuhan University, Wuhan, China, 430072 \\ \{yxli@whu.edu.cn, wangww@mail.whut.edu.cn, weiqinying@yahoo.com.cn\} \\ ${ }^{2}$ Department of Computer Science \\ Central China Normal University, Wuhan, China, 430079 \\ xjshen@mail.ccnu.edu.cn \\ ${ }^{3}$ College of Computer Science \\ South-Central University For Nationalities, Wuhan, China, 430074 \\ zhengbojin@gmail.com
}

\begin{abstract}
It is recognized that evolutionary algorithms are inspired from evolutionary biology. In this paper, we set up a thermodynamic model of evolutionary algorithm. This model is intuitive and has a solid foundation in thermodynamics. It is our first step towards a unified theory of evolutionary algorithms.
\end{abstract}

Keywords: Evolutionary Algorithm, Thermodynamics, Thermodynamic Model of Evolutionary Algorithm.

\section{Introduction}

The term Evolutionary Algorithm (EA) stands for a family of stochastic problem solvers based on principles that can be found in biological evolution. Within this paradigm, achieving a solution to a given problem is seen as a survival task: possible solutions compete with the others for survival, and this competition is the driving force behind the progress that supposedly leads to an optimal solution [1].

There are numerous successful applications of EA in business and industry, but such successes are not fully understood. It has been proposed a lot of theories such as schema theory [2, Markov chains theory [3], dimensional analysis 4, order statistics [5], quantitative genetics [6], quadratic dynamical systems [7] and statistical physics [8]. But the problem of these theories is either that they are too complicated to understand or they do not apply to algorithms that are widely used. Is there an intuitive and unified theory of EA? In this paper, we present our preliminary result of this research. It is the thermodynamic model of EA.

\section{Basis of Thermodynamics}

Thermodynamics is a branch of physics which deals with the energy and work of a system. Thermodynamics deals only with the large scale response of the 
system. Small scale interactions are described by kinetic theory. These both theories compliment the other; some principles are more easily understood in terms of thermodynamics and some other principles are more easily explained by kinetic theory. The association between thermodynamics and kinetic theory can be derived by statistical mechanics.

\subsection{Thermodynamic System}

A thermodynamic system is a quantity of matter of fixed identity, around which we can draw a boundary. The boundaries may be fixed or moveable. Work or heat can be transferred across the system boundary. Everything outside the boundary is called the surroundings.

\subsection{The Zeroth Law}

The zeroth law of thermodynamics begins with a simple definition of thermodynamic equilibrium. Thermodynamic equilibrium can be observed as follow: if two of these objects are brought into physical contact there is initially a change in the property of both objects, but, eventually, the change in property stops. The objects are said to be in thermodynamic equilibrium.

\subsection{The First Law}

The first law of thermodynamics is the application of the conservation of energy principle to heat and thermodynamic processes. Any thermodynamic system in an equilibrium state possesses a state variable called the internal energy. Between any two equilibrium states, the change in internal energy is equal to the difference of heat transfer into the system and work done by the system.

There are three ways that heat may be transferred between substances at different temperatures: conduction, convection, and radiation. The flow of heat by conduction occurs via collisions between molecules in the substance and the subsequent transfer of translational kinetic energy. Convection is the flow of heat through a bulk, macroscopic movement of matter from a hot region to a cool region, as opposed to the microscopic transfer of heat between molecules involved with conduction. The third and last form of heat transfer is radiation, which in this context means light. For example, from the sun to the earth through mostly empty space - such a transfer cannot occur via convection nor conduction, which require the movement of material from one place to another or the collisions of molecules within the material. From above, the change of internal energy is caused by the changes of micro states of molecules.

\subsection{The Second Law}

The first law of thermodynamics allows for many possible states of a system to exist. But, experience indicates that only certain states occur. This leads to the second law of thermodynamics and the definition of another state variable called 
entropy. Entropy can be defined as a measure of the multiplicity of a system. For a system of a large number of molecules, like a mole of molecules, the most probable state will be found in the state of highest multiplicity. Accordingly, the distribution at the time is the maximum entropy distribution.

\section{Thermodynamic Model of Evolutionary Algorithm}

There are many similarities between thermodynamic system and EA population. Firstly, a thermodynamic system is made up by a number of molecules, while the EA population is also made up by a number of individuals. Secondly both the molecules and individuals work randomly.

In the thermodynamic model of EA, a population is a thermodynamic system. An individual is a molecule. The chromosome of the individual presents the micro state of the molecule. The energy of the molecule is the fitness of the individual, which is decided by the problem. The sum of all individual fitness is the fitness of the population. It is the internal energy of the system.

For a minimization problem, the task is to find the micro state of the molecule with the lowest energy, which is referred as the ground state. For a given micro state, it is easy to assign an energy, but it is hard to construct a micro state for a given energy. The way to find the ground state is using the search operator.

There are two kinds of search operators in EA, especially for GA. One is mutation. The other is crossover. In the thermodynamic model of EA, mutation is similar to radiation and crossover is similar to conduction. Both mutation and crossover are means of heat transferring.

The survival strategy and update strategy in the thermodynamic model of EA are the applications of the conservation of energy principle and the conservation of mass principle. Before the application of search operator, the parents, who are selected by selection operator according to the distribution of individuals, possess some amount of energy. After the application of search operator, the children possess other amount of energy. The survivals are the whole of parent or the whole of children who possess less energy. The winers take place of the losers. The replacement leads to a new distribution of individuals and a new state of the system. The change of system state leads to the change of system energy. The change of system energy is the work done by the algorithm.

If the given time of running is long enough, the population and algorithm get into thermodynamic equilibrium. We can analyze the property, performance and efficiency of the algorithm by the termination state of the population.

The summary of thermodynamic model of evolutionary algorithm is show in Tab11.

\section{Future Work}

In this paper, we set up a thermodynamic model of EA. It has a solid foundation in thermodynamics. It can be taken as a reference model of EA to compare the 
Table 1. Summary of thermodynamic model of evolutionary algorithm

\begin{tabular}{|c|c|}
\hline EA & Thermodynamics \\
\hline population & system \\
\hline individual & molecule \\
\hline chromosome & micro state \\
\hline fitness function & energy function \\
\hline individual fitness & molecular energy \\
\hline population fitness & internal energy \\
\hline selection & distribution \\
\hline crossover & conduction \\
\hline mutation & radiation \\
\hline optimal solution & ground state \\
\hline
\end{tabular}

difference between EAs. In the future, we hope we can propose a unified theory of EA based on the thermodynamic model of EA.

\section{Acknowledgment}

This paper was supported by the National Natural Science Foundation of China under Grant No.60473014, and the Specialized Research Fund for the Doctoral Program of Higher Education of China under Grant No.20030486049.

\section{References}

1. Eiben, A.E., Rudolph, G.: Theory of evolutionary algorithms: a bird's eye view. Theoretical Computer Science 229(1-2) (1999) 3-9

2. Holland, J.: Adaptation in Natural and Artificial Systems. University of Michigan Press, Ann Arbor, USA (1975)

3. Nix, A.E., Vose, M.D.: Modeling genetic algorithms with markov chains. Annals of Mathematics and Artificial Intelligence 5(1) (1992) 79-88

4. Goldberg, D., Deb, K., Thierens, D.: Toward a better understanding of mixing in genetic algorithms. Journal of the Society for Instrumentation and Control Engeering 32(1) (1993) 10-16

5. Beyer, H.G.: Toward a theory of evolution strategies: the $(\mu, \lambda)$-theory. Evolutionary Computation 2(4) (1994) 381-407

6. Mühlenbein, H., Schlierkamp-Voosen, D.: Predictive models for the breeder genetic algorithm I: Continuous parameter optimization. Evolutionary Computation 1(1) (1993) 25-49

7. Vose, M.D., Liepins, G.E.: Punctuated equilibria in genetic search. Complex Systems 5(1) (1991) 31-44

8. Prügel-Bennett, A., Shapiro, J.L.: An analysis of genetic algorithms using statistical mechanics. Physical Review Letters 72 (1994) 1305-1309 